\title{
PERTINENCIA DE LOS PROGRAMAS SOCIALES EN EL ESTADO DE MÉXICO DIRIGIDOS A SU POBLACIÓN ENVEJECIDA
}

\author{
RELEVANCE OF SOCIAL PROGRAMS FOR THE AGING IN THE ESTADO DE MÉXICO
}

\author{
Zoraida Ronzón Hernández \\ Centro de Investigación y Estudios en Ciencias Sociales y Humanidades \\ Universidad Autónoma del Estado de México \\ zoraronzon@hotmail.com \\ Pablo Jasso Salas \\ Facultad de Antropología, Universidad Autónoma del Estado de México \\ pablo_jasso@hotmail.com
}

\section{RESUMEN}

Este trabajo pretende mostrar si las políticas públicas vigentes en el Estado de México respecto de la población envejecida responden a las necesidades percibidas por ese sector. Se basa en un trabajo etnográfico realizado en 12 municipios de la entidad, poniendo énfasis en la manera en que personas mayores de 60 años perciben la eficacia y pertinencia de los programas sociales, tanto de transferencia económica como en especie, cómo inciden en su vida cotidiana y si contribuyen a su estabilidad, y por otro lado muestra los «deseos» o necesidades de esta población y sus críticas a la manera en que se asignan actualmente los apoyos.

PALABRAS CLAVE: envejecimiento, percepción, programas sociales, Estado de México.

\section{ABSTRACT}

This article aims to demonstrate whether the public policies in effect in the Estado de México regarding the aging population do respond to the needs perceived by this sector of individuals. It is based on ethnographic work conducted in 12 municipalities, highlighting the way in which people aged over 60 perceive the effectiveness and relevance of social programs that carry out economic or in-kind support, how this impacts their everyday life and whether or not this contributes to their stability. It also reflects their «desires» or needs and their criticisms regarding the way in which this support is allocated.

KEY WORDS: aging, perception, social programs, Estado de México. 


\section{INTRODUCCIÓN}

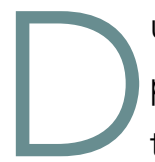

urante las últimas dos décadas, dentro de los trabajos dedicados a la población envejecida, se ha desarrollado una gama importante de temáticas en torno a ese sector, desde la base de los estudios demográficos hasta los antropológicos sobre subjetividad y percepción; sin embargo, muchos de estos se han quedado en anaqueles de bibliotecas o viviendo en los mundos virtuales de las nuevas tecnologías de la información, conocidas mayormente como TIC (en el mejor de los casos), y otros más han quedado perdidos dentro de archivos personales (físicos o virtuales, eso es lo de menos). En este punto los investigadores sociales se cuestionan la utilidad de esos años de investigación y sus resultados, incluso se llega a la discusión de la actividad intelectual misma; sin embargo, 20 años después de que en México se empezara a hacer investigación con respecto a la población envejecida, el tema se ha vuelto objeto de discusión más allá de la academia.

Este trabajo pretende mostrar si en el Estado de México las políticas públicas destinadas a la población envejecida corresponden o no a las necesidades que este grupo de edad manifiesta, y plantear que las ciencias sociales, en concreto la antropología, debieran intentar tener injerencia en las políticas públicas como lo hicieron hace más de medio siglo Gonzalo Aguirre Beltrán (1955, 1973), Alfonso Caso (1948) o Guillermo Bonfil Batalla (1991), entre otros, en lo tocante a las problemáticas de salud, educación o atención a la población indígena, con eco no solo en el contexto estatal sino en el conjunto de la sociedad.

La investigación implicaba el trabajo con diferentes variables que permean el desarrollo y la estabilidad de este grupo poblacional: por una parte, la diferenciación entre población urbana y rural, bajo el supuesto de que la pobreza se concentra en localidades pequeñas; y por otra, dos variables indispensables son la familia y la seguridad social; ambos elementos son determinantes para considerar si la etapa es o no estable en cuanto a la cobertura de necesidades elementales, entre las cuales encontramos la alimentación, el vestido y la salud.

Conviene recordar aquí que la antropología tiene la intención de entender y explicar la manera en que el ser humano vive y reproduce la cultura. Se utilizan métodos y técnicas (cualitativas, por supuesto) que ayudan a desentrañar la multiplicidad de hechos sociales en que participa cotidianamente el sujeto. Dado lo anterior, la antropología aplicada pretendería, entonces, utilizar el resultado de un trabajo con la premisa de que el investigador se inserte en una determinada 
problemática social, de manera que el problema de investigación adquiera propiedades complejas que implican buscar una solución. Foster (1974), por ejemplo, hace evidente que la antropología aplicada tiene la intención planificada de intervenir, y afirma que debe tener dos cualidades: utilizar teoría propia de la disciplina y que esta sea llevada a la práctica.

El dilema de la articulación entre la teoría y la práctica radica, precisamente, en la formación básica del antropólogo, que nos ha enseñado a No intervenir en la realidad observada, por lo que la dificultad más importante se remite a la aplicación de la antropología misma; y por otro lado se encuentra el hecho de que en una investigación se utilizan conceptos de diferentes perspectivas teóricas, que si bien explican y articulan el problema, por otro lado, «pueden remitir a investigaciones empíricas, pero frecuentemente los que los producen no hacen investigaciones sobre la 'realidad' o, si se prefiere, sobre 'problemas' de la realidad, sino que lo que hacen es reflexionar sobre cómo es descrita y sobre todo cómo es analizada la realidad» (Menéndez 1999:149), y es quizás este el problema de las políticas públicas vigentes en el Estado de México, en torno a la población envejecida, donde pareciera que los programas sociales existentes han partido de la teorización y terminan teniendo una falta de practicidad, debido seguramente a que no están basados en trabajos que observen directamente la realidad social.

\section{LA INVESTIGACIÓN EMPÍRICA}

Por sus características, el Estado de México es por sí mismo trascendente dentro del proceso de envejecimiento demográfico en el país; por ejemplo, teniendo solo $1.1 \%$ del territorio nacional, cuenta con más de 15 millones de habitantes, lo que equivale a $13.5 \%$ de la población total. De hecho, es el estado más poblado. Ahora bien, es importante destacar que 1137647 habitantes en esa entidad, alrededor de 8\%, son adultos mayores (Conapo 2012).

En cuanto a su distribución, la entidad está conformada por 125 municipios (Secretaría de Finanzas y Planeación 1995, CEDEMUN 1997). Casi una tercera parte del estado pertenece a dos grandes zonas metropolitanas: la de la Ciudad de México (21 municipios) y la del Valle de Toluca (14 municipios), ${ }^{1}$ de manera que $87 \%$ de su población vive en zonas urbanas, y solo $13 \%$ en áreas rurales,

1 De ahora en adelante, para hablar de las dos zonas metropolitanas se utilizará ZMCM para la de la Ciudad de México y ZMVT para la del Valle de Toluca. 
superando así la proporción nacional, que es de $78 \%$ y $22 \%$, respectivamente (INEGI 2011).

La pluralidad de la población mexiquense se hace evidente en los grupos indígenas establecidos en la entidad, aunque los hablantes de alguna lengua indígena reportados por el INEGI en el censo del 2010 sean solamente 2\%, por debajo del $6 \%$ nacional.

El Estado de México aporta 9.2\% del PIB del país, principalmente de su actividad industrial; sin embargo, sigue manteniendo una producción agrícola.

En este contexto se desenvuelve la población de más de 60 años que, en el Estado de México, vive las consecuencias de la transición demográfica. Durante el trabajo de campo que da sustento a este artículo se investigó en 12 municipios. Se realizó trabajo etnográfico y se recopilaron narrativas autobiográficas, ya que recuperan elementos de la historia de vida desde la voz del informante, y así se evidencia su subjetividad y sus valoraciones desde la perspectiva de sus necesidades y sus percepciones sobre los programas sociales.

Realizar trabajos de investigación desde la antropología implica, como dice Vázquez, "percibir, interpretar y comprender el sentido que los ancianos dan a su vida, la forma en que la asumen diariamente [y] cómo esta forma de asumirla se modifica al llegar a la vejez» (1999:70). Reyes (1999:8), por su parte, afirma que existe una necesidad de estudios más globales, más allá de los puramente demográficos basados en datos cuantitativos, para poder comprender la ancianidad y el envejecimiento como el resultado de un proceso que se ha gestado en diversas etapas del ciclo de vida. De ahí que este trabajo combine tanto métodos cuantitativos como cualitativos para fortalecer la investigación.

Se aplicaron entrevistas estructuradas, semiestructuradas, abiertas, y se obtuvo información por observación directa y consultando fuentes de datos cualitativos en torno a las políticas y los programas sociales a nivel estatal; sin embargo, también se utilizó metodología cuantitativa para determinar los municipios en los que se realizaría el trabajo de campo propiamente dicho.

La elección de los 12 municipios para la investigación respondió a un diagnóstico cuantitativo previo basado en el Censo de población y vivienda 2010 (INEGI 2011), se consideraron los más adecuados o de los que era necesario obtener información, bajo los siguientes parámetros (mapa 1):

Municipios rurales y/o con población indígena: Temascalcingo (población Mazahua), Temoaya (donde se ubica el Centro Ceremonial Otomí), Tejupilco (con 
población nahua), Amatepec, Coatepec Harinas y Tonatico; estos tres últimos presentan un alto índice de envejecimiento.

Municipios urbanos-metropolizados: Cuautitlán Izcalli, Tlalnepantla de Baz, Ixtapaluca y Valle de Chalco (pertenecientes a la ZMCM), los dos primeros con la mayor concentración de población de más de 60 años, y los dos últimos con el más bajo porcentaje de población envejecida. De la ZMVT se trabajaron Toluca y Metepec, debido a que presentaron la más alta cobertura de derechohabientes por alguna institución de seguridad social y de salud.

Mapa 1. Distribución de los municipios abordados en la investigación. Fuente: Elaboración propia con información de INEGI, 2011.

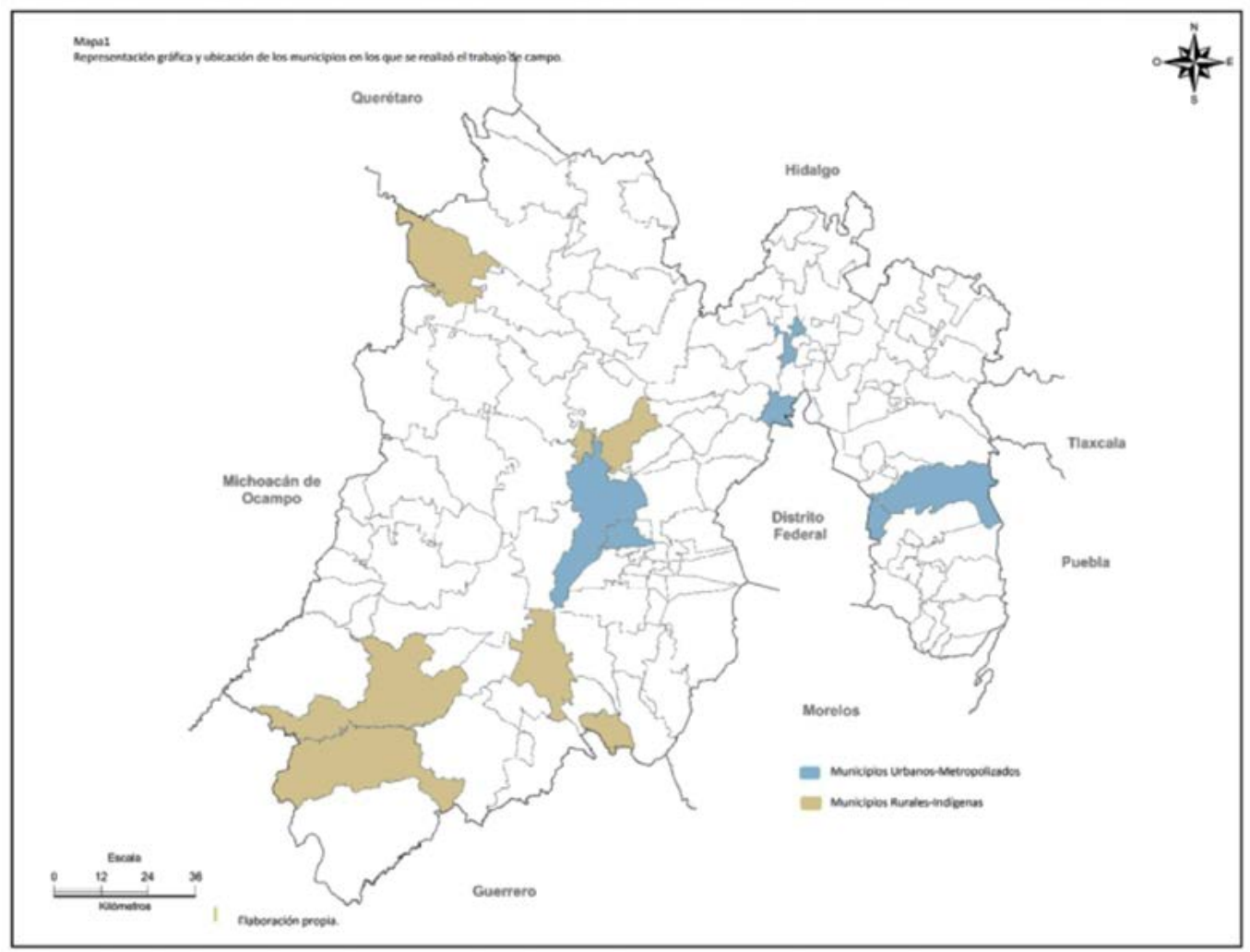

Se realizaron un total de 48 entrevistas, cuatro en cada municipio, con la intención de obtener las narrativas autobiográficas.

- Los criterios de inclusión para las entrevistas fueron:

- Que tuvieran más de 60 años de edad

- Dos hombres y dos mujeres por municipio 
- Un hombre y una mujer con seguridad social

- Un hombre y una mujer sin seguridad social

Como información general de los sujetos del estudio se tienen los siguientes resultados:

- Tienen entre 62 y 81 años de edad

- Solo una persona del sexo femenino no tuvo hijos

- El número de hijos va de los 3 a los 12

- El número de nietos está entre los 2 y los 26

- Se pueden encontrar hasta bisnietos y una persona tiene un tataranieto.

- La escolaridad oscila entre el saber escribir solo su nombre, con ningún año escolar cursado, hasta estudios de licenciatura.

- El grupo de viejos se muestra, así, como un conjunto heterogéneo.

\section{LOS PROGRAMAS PÚBLICOS DEL ESTADO DE MÉXICO}

Las políticas públicas por sí mismas no han sido objeto preferencial de estudio desde la antropología; sin embargo, los antecedentes se asientan en la antropología política, y nos ponen ante un panorama social contemporáneo donde resulta indudable la necesidad de observar y evidenciar las medidas en materia económica, política y social que se han desarrollado alrededor de la población envejecida, en este caso, en el Estado de México, con la finalidad de incrementar en el futuro la atención de las demandas de salud, educación, infraestructura y seguridad social para poder garantizar una vida digna y segura a las siguientes generaciones que envejecerán. Es necesario investigar alrededor de las políticas sociales que desarrolla el Estado para hacer frente a las desigualdades y desventajas de las personas de más de 60 años; resulta, entonces, primordial clarificar cómo se entiende el término «política social», ya que este evoca como idea central, según López (2004:30), un espacio de intervención estatal, es decir, extraeconómica, orientada a la asignación legítima de recursos entre clases, grupos e individuos, cuyo resultado es la promoción o exclusión, de manera que una política social designará a beneficiarios o destinatarios de recursos — beneficiosdispuestos por el aparato estatal.

La situación cada vez más crítica de esta población provocó que en años recientes se pusieran en operación programas públicos para atenderla, los cuales, 
como se tratará de mostrar, no deben concentrarse solo en la provisión de recursos monetarios o dotación de apoyos en especie que parecieran «beneficios» para este sector, de lo que las burocracias han teorizado sobre las necesidades cotidianas de la población de 60 años y más, sino que además deben atender las necesidades que los interesados manifiestan.

Aunque los problemas sociales tienen que ver con situaciones que se viven individualmente, su solución requiere considerarlos como problemas poblacionales, que no son la sumatoria de las características de los individuos y sí el resultado de sus interacciones y su ubicación en la estructura social, ya que la política social del Estado tiene que ir encaminada al bienestar de la población (Ávalos 2005), por lo que las acciones desarrolladas por las distintas instituciones deben poner énfasis en las principales necesidades de la población hacia la cual se dirigen las políticas.

En el Estado de México los programas sociales y asistenciales que atienden a la población envejecida están a cargo del Sistema para el Desarrollo Integral de la Familia (DIF), dentro del Comité Estatal para la Atención del Adulto Mayor, donde en su programa 2010 consistía en grupos de trabajo de:

- Asistencia social

- Salud

- Educación

- Publicidad y medios de comunicación

- Trabajo y seguridad social: capacitaciones, promoción, prevención y protección a la salud, asesorías jurídicas, incorporación al trabajo remunerado, círculos de convivencia.

A continuación (cuadro 1) se presentan los programas vigentes para la actual administración que se dirigen o que de alguna manera incluye a la población envejecida:

Cuadro 1. Programas públicos para la atención de la población de más de 60 años.

\begin{tabular}{|c|c|c|c|}
\hline Programa & Tipo de apoyo & $\begin{array}{l}\text { Institución que } \\
\text { administra }\end{array}$ & $\begin{array}{l}\text { Población } \\
\text { beneficiada }\end{array}$ \\
\hline $\begin{array}{l}\text { Programa } \\
\text { de unidades } \\
\text { móviles } \\
\text { médico- } \\
\text { odontológicas }\end{array}$ & $\begin{array}{l}\text { Atención médico-odontológica } \\
\text { preventiva y curativa gratuita. } \\
\text { Entrega de medicamento a la } \\
\text { población carente de asistencia } \\
\text { social en las comunidades rura- } \\
\text { les y suburbanas. }\end{array}$ & $\begin{array}{l}\text { Sistemas } \\
\text { Municipales DIF, } \\
\text { instituciones } \\
\text { gubernamentales } \\
\text { y/o asociaciones } \\
\text { civiles }\end{array}$ & $\begin{array}{l}\text { Adultos mayores y } \\
\text { población que no } \\
\text { cuente con segu- } \\
\text { ridad social en las } \\
\text { comunidades rura- } \\
\text { les y suburbanas. }\end{array}$ \\
\hline
\end{tabular}




\begin{tabular}{|c|c|c|c|}
\hline Programa & Tipo de apoyo & $\begin{array}{l}\text { Institución que } \\
\text { administra }\end{array}$ & $\begin{array}{l}\text { Población } \\
\text { beneficiada }\end{array}$ \\
\hline $\begin{array}{l}\text { Integración de } \\
\text { grupos de adul- } \\
\text { tos mayores }\end{array}$ & $\begin{array}{l}\text { Convivencia con otros adultos } \\
\text { mayores o diferentes actividades } \\
\text { recreativas. }\end{array}$ & $\begin{array}{l}\text { Coordinación de } \\
\text { Atención a Adultos } \\
\text { Mayores }\end{array}$ & Adultos mayores \\
\hline $\begin{array}{l}\text { Capacitación } \\
\text { a adultos ma- } \\
\text { yores }\end{array}$ & $\begin{array}{l}\text { Talleres de capacitación y becas } \\
\text { para los adultos mayores en es- } \\
\text { pecialidades de corte y confec- } \\
\text { ción industrial, cultura de belle- } \\
\text { za, computación, reparación de } \\
\text { aparatos electrodomésticos y } \\
\text { pintura textil. }\end{array}$ & $\begin{array}{l}\text { Secretaría del } \\
\text { Trabajo }\end{array}$ & Adultos mayores \\
\hline $\begin{array}{l}\text { Credencial } \\
\text { DIFEM para } \\
\text { Adultos } \\
\text { Mayores }\end{array}$ & $\begin{array}{l}\text { Permite acceder a descuentos } \\
\text { en compras o al pagar un servi- } \\
\text { cio en los establecimientos con } \\
\text { los que la Institución o los Siste- } \\
\text { mas Municipales DIF, tienen cele- } \\
\text { brado convenio. }\end{array}$ & $\begin{array}{l}\text { DIF Estado de } \\
\text { México }\end{array}$ & Adultos mayores \\
\hline $\begin{array}{l}\text { Programa de } \\
\text { Desarrollo } \\
\text { Social Gente } \\
\text { Grande Ver- } \\
\text { tiente Adultos } \\
\text { Mayores de } 60 \\
\text { a } 69 \text { años de } \\
\text { edad }\end{array}$ & $\begin{array}{l}\text { Canasta alimentaria de manera } \\
\text { mensual. }\end{array}$ & $\begin{array}{l}\text { Consejo Estatal de } \\
\text { la Mujer y Bienestar } \\
\text { Social }\end{array}$ & $\begin{array}{l}\text { A los adultos de } 60 \\
\text { a } 69 \text { años de edad }\end{array}$ \\
\hline $\begin{array}{l}\text { Trabajo Social } \\
\text { para Adultos } \\
\text { Mayores }\end{array}$ & $\begin{array}{l}\text { Realización de estudios socioe- } \\
\text { conómicos y asesoría ante pro- } \\
\text { blemáticas familiares y de salud } \\
\text { brindando alternativas de solu- } \\
\text { ción. }\end{array}$ & $\begin{array}{l}\text { Sistema de } \\
\text { desarrollo integral } \\
\text { para la familia } \\
\text { Gobierno del } \\
\text { Estado de México }\end{array}$ & $\begin{array}{l}\text { Estudiantes y/o } \\
\text { trabajadores o } \\
\text { voluntarios }\end{array}$ \\
\hline $\begin{array}{l}\text { Casa del Adulto } \\
\text { Mayor }\end{array}$ & $\begin{array}{l}\text { Espacio de recreación, conviven- } \\
\text { cia y capacitación, con talleres } \\
\text { y actividades que se imparten } \\
\text { como: danza regional, baile de } \\
\text { salón, yoga, taichi, cocina, ma- } \\
\text { nualidades, acondicionamiento } \\
\text { físico y alfabetización. }\end{array}$ & $\begin{array}{l}\text { Coordinación de } \\
\text { Atención a Adultos } \\
\text { Mayores }\end{array}$ & Adultos mayores \\
\hline $\begin{array}{l}\text { Control de Cali- } \\
\text { dad y Nutrición }\end{array}$ & $\begin{array}{l}\text { Pretende seleccionar, evaluar y } \\
\text { dar y seguimiento a la calidad de } \\
\text { los productos que forman parte } \\
\text { de la integración de las canastas } \\
\text { alimentarias de los diferentes } \\
\text { programas. }\end{array}$ & $\begin{array}{l}\text { Consejo Estatal de } \\
\text { la Mujer y Bienestar } \\
\text { Social }\end{array}$ & $\begin{array}{l}\text { Adultos mayores } \\
\text { y mujeres } \\
\text { embarazadas }\end{array}$ \\
\hline $\begin{array}{l}\text { Tarjeta Com- } \\
\text { promiso }\end{array}$ & $\begin{array}{l}\text { Con ella se reciben descuen- } \\
\text { tos en bienes y servicios en los } \\
\text { más de } 3000 \text { establecimientos } \\
\text { afiliados al programa que están } \\
\text { ubicados en todo el territorio es- } \\
\text { tatal. Dichos descuentos oscilan } \\
\text { entre } 5 \% \text { y } 100 \% \text {, abarcando la } \\
\text { salud, alimentación, turismo, en- } \\
\text { tretenimiento, entre otros. }\end{array}$ & $\begin{array}{l}\text { Consejo Estatal de } \\
\text { la Mujer y Bienestar } \\
\text { Social }\end{array}$ & $\begin{array}{l}\text { Adultos mayores } \\
\text { que sean } \\
\text { beneficiarios de uno } \\
\text { de los programas de } \\
\text { pensión alimenticia } \\
\text { del CEMyBS }\end{array}$ \\
\hline
\end{tabular}




\begin{tabular}{|c|c|c|c|}
\hline Programa & Tipo de apoyo & $\begin{array}{l}\text { Institución que } \\
\text { administra }\end{array}$ & $\begin{array}{l}\text { Población } \\
\text { beneficiada }\end{array}$ \\
\hline Tarjeta INAPAM & $\begin{array}{l}\text { Se le reconoce como adulto ma- } \\
\text { yor y merecedor de los progra- } \\
\text { mas sociales destinados a esta } \\
\text { población. }\end{array}$ & $\begin{array}{l}\text { INAPAM (Instituto } \\
\text { Nacional de las } \\
\text { Personas Adultas } \\
\text { Mayores }\end{array}$ & Adultos mayores \\
\hline $\begin{array}{l}\text { Programa de } \\
\text { Desarrollo } \\
\text { Social Gente } \\
\text { Grande ver- } \\
\text { tiente Adultos } \\
\text { Mayores de } 70 \\
\text { años de edad }\end{array}$ & $\begin{array}{l}\text { Favorecer el acceso a alimentos } \\
\text { en personas de } 70 \text { años de edad } \\
\text { o más, que viven en condición } \\
\text { de pobreza multidimensional en } \\
\text { el Estado de México, a través del } \\
\text { otorgamiento de una canasta ali- } \\
\text { mentaria, además de artículos } \\
\text { de aseo personal y de limpieza. }\end{array}$ & $\begin{array}{l}\text { Consejo Estatal de } \\
\text { la Mujer y Bienestar } \\
\text { Social }\end{array}$ & $\begin{array}{l}\text { A los adultos mayo- } \\
\text { res de } 70 \text { años de } \\
\text { edad en adelante. }\end{array}$ \\
\hline $\begin{array}{l}\text { Convivencia In- } \\
\text { tergeneracional }\end{array}$ & $\begin{array}{l}\text { Convivencias entre adultos ma- } \\
\text { yores de diferentes municipios } \\
\text { con menores de los albergues } \\
\text { del DIFEM, así como con adultos } \\
\text { mayores de otros municipios. } \\
\text { Pretende promover el respeto } \\
\text { a los adultos mayores haciendo } \\
\text { de ellos difusores de la indepen- } \\
\text { dencia y los valores patrios. }\end{array}$ & $\begin{array}{l}\text { DIF Estado de } \\
\text { México }\end{array}$ & Adultos mayores \\
\hline $\begin{array}{l}\text { Paseos recrea- } \\
\text { tivos para adul- } \\
\text { tos mayores }\end{array}$ & $\begin{array}{l}\text { Pasear y conocer diferentes lu- } \\
\text { gares de la entidad. }\end{array}$ & $\begin{array}{l}\text { Coordinación de } \\
\text { Atención a Adultos } \\
\text { Mayores. }\end{array}$ & Adultos mayores \\
\hline $\begin{array}{l}\text { Consulta } \\
\text { médica y } \\
\text { asesoría jurídica } \\
\text { para adultos } \\
\text { mayores }\end{array}$ & $\begin{array}{l}\text { Cuenta con un médico general } \\
\text { en la Casa del Adulto Mayor } \\
\text { para dar consulta en caso de } \\
\text { requerirse; para problemas de } \\
\text { tipo legal tiene asesoría jurídica, } \\
\text { y si el caso lo requiere se cana- } \\
\text { liza a las instancias competentes } \\
\text { de acuerdo a la naturaleza del } \\
\text { asunto como Instituto de la De- } \\
\text { fensoría de Oficio, Colegio de } \\
\text { Notarios, Dirección de Servicios } \\
\text { Jurídico Asistenciales del DIFEM, } \\
\text { entre otros. }\end{array}$ & $\begin{array}{l}\text { Sistema de } \\
\text { Desarrollo Integral } \\
\text { para la Familia } \\
\text { Gobierno Estado } \\
\text { de México }\end{array}$ & Adultos mayores \\
\hline
\end{tabular}

Fuente: Elaboración propia con información del Gobierno del Estado de México.

El cuadro 1 muestra que todos de los programas sociales destinados a la población de 60 años y más en el Estado de México son meramente asistencialistas ${ }^{3}$ y ninguno se enfoca a satisfacer, y mucho menos a solucionar problemáticas en

2 Información obtenida de las páginas del Gobierno del Estado y del DIF Estatal durante los meses de septiembre a noviembre de 2014.

3 Se entiende por asistencialismo la manera en que el Estado realiza acciones encaminadas al otorgamiento de bienes pero no a la construcción de políticas públicas que contribuyan a la solución de los problemas reales. Aranibar (2001:8) dice que el asistencialismo implica «desde conside- 
torno a este sector, y que se desarrollan por parte de diferentes instituciones gubernamentales, lo que denota la falta de un organismo dedicado exclusivamente a la atención de personas mayores de 60 años.

El DIF mantiene el Programa Nacional de Atención Integral para los Adultos Mayores, cuyo objetivo es «normar, coordinar, promover e instrumentar acciones que tengan como finalidad mejorar el nivel y la calidad de vida de la población adulta mayor del país, fortalecer sus capacidades y garantizar sus derechos humanos a través de los servicios que presta el DIF». Se pretende brindar atención integral a esta población, poner a su alcance servicios asistenciales que contribuyan a su integración y desarrollo individual, social, cultural y recreativo, promover su reintegración al núcleo familiar o social, proporcionarles apoyos económicos, alimenticios o en especie, considerando sus condiciones de pobreza, marginación y vulnerabilidad; ofrecer capacitación gerontológica continua al personal que tiene contacto con ella y promover su reconocimiento social, económico y político.

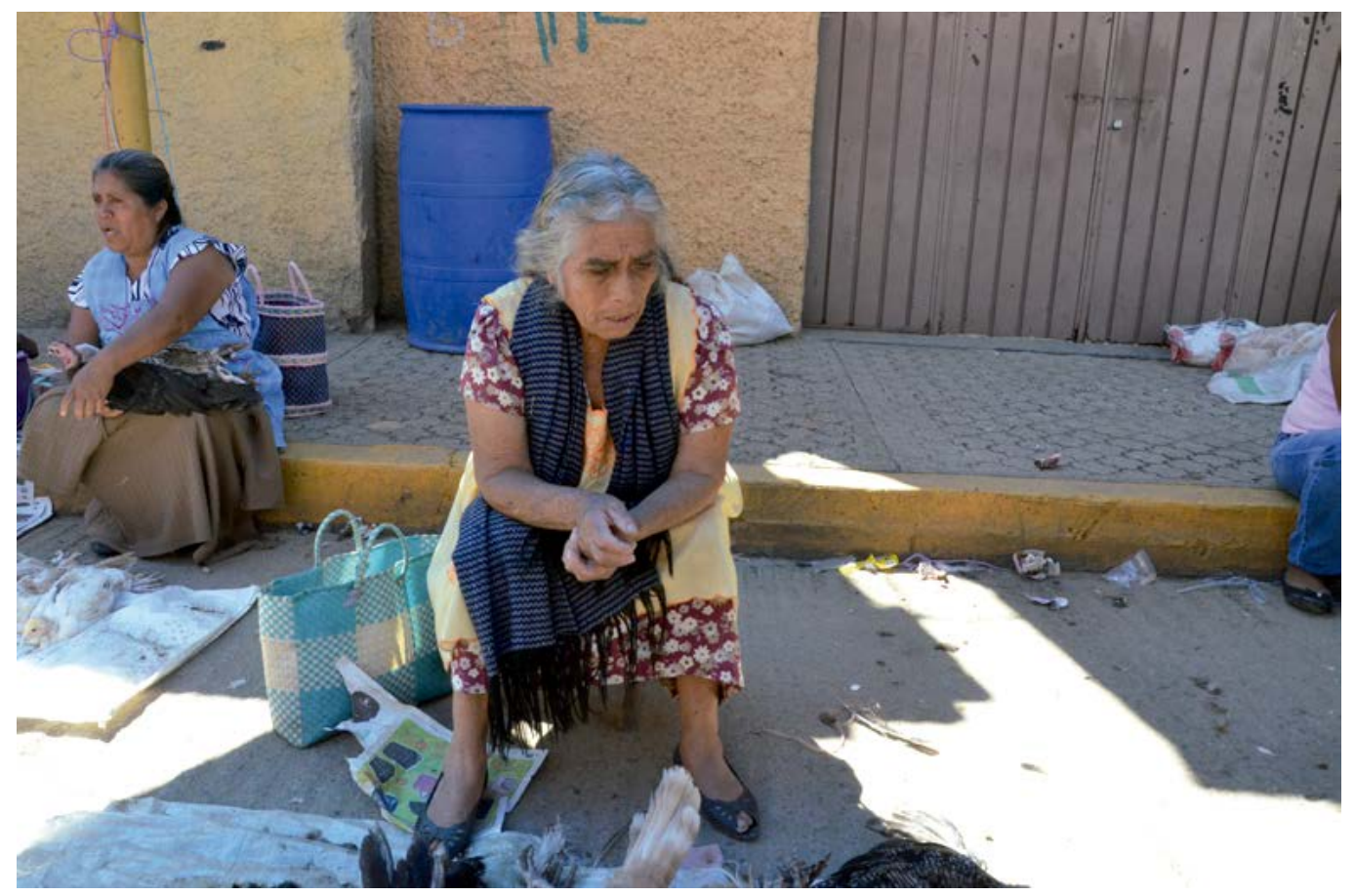

Fotografías de Pablo Jasso Salas.

rar solo la asistencia directa en insumos básicos... la asistencia como método de intervención, a la que se denominará asistencialismo». 
La Secretaria de Desarrollo Social (Sedesol) establece un Programa de Atención a Adultos Mayores en Zonas Rurales cuyo objetivo es apoyar a los adultos mayores en condiciones de pobreza alimentaria que no son atendidos por algún programa federal y que viven en localidades rurales con menos de 2500 habitantes, de alta y muy alta marginación, haciendo una transferencia a su ingreso mediante apoyo económico.

El Instituto de Salud del Estado de México, por medio de la Secretaría de Salud, cuenta con un programa cuyo objetivo es prevenir, controlar y retardar la presencia de las enfermedades discapacitantes y la muerte, elevar la calidad de vida de los adultos mayores y alcanzar una cultura a favor del envejecimiento. Plantea acciones estratégicas para identificar problemas de salud más frecuentes a través de la aplicación de encuestas; promover la utilización de la Cartilla de Salud del Adulto Mayor y la aplicación de vacunas; desarrollar con periodicidad anual la Semana de Salud para Gente Grande; incorporar al adulto mayor hipertenso al plan para la reducción de la mortalidad por enfermedad cerebrovascular e incorporar a los adultos mayores a los grupos de ayuda mutua y continuar la operatividad del Comité Estatal de Atención al Envejecimiento.

Debe mencionarse que todos estos programas estatales tienen como marco de referencia los programas federales de Atención a los Adultos Mayores de 70 años y más en Zonas Rurales de Sedesol, ${ }^{4}$ que prestan apoyo económico e impulsan la generación de una red de protección social. Se atiende a aquellos que vivan en localidades de hasta 30000 habitantes. Los beneficiarios reciben apoyos económicos de 500 pesos mensuales, que se pagan cada dos meses; participan en grupos de convivencia y actividades informativas sobre temas de salud y obtienen facilidades para el acceso a servicios y apoyos de instituciones como el INAPAM, además de las que ofrecen actividades productivas y ocupacionales.

\section{EL ESTADO DE MÉXICO Y LAS NECESIDADES DE SU POBLACIÓN ENVEJECIDA}

El Estado de México es una entidad que ha mantenido, a lo largo de las últimas dos décadas, un creciente y progresivo número de personas de más de 60 años de edad, lo que hace que sea una sociedad en proceso de envejecimiento, y que lleva una serie de necesidades sociales, económicas, políticas y culturales, por

${ }^{4}$ Es importante resaltar que este trabajo se realizó durante el periodo comprendido entre 2013 y 2014, por lo que no había entrado en vigor la reestructuración al programa que cambió la edad mínima a 65 años, aunque por lo demás sigue aplicándose en los mismos términos. 
lo cual la seguridad social resulta de vital importancia para atender a la población; sin embargo, alrededor de esto se encuentra una gama de problemas que dificultan no solo la aplicación de políticas públicas adecuadas sino también la observación misma de la realidad.

En relación a esto se encuentra la ocupación de las personas de más de 60 años de edad en la entidad, teniendo como base las actividades laborales que desarrollaron a lo largo de su vida, donde el principal sector en el que se desempeñaron laboralmente fue el sector de los servicios, provocado por el contexto histórico específico que vivió este grupo etario, por lo que es importante resaltar que, como dice Martínez, ha quedado documentado que en lo que se refiere al sector terciario que «algunos efectos de la reestructuración económica tales como la contratación del mercado interno debido a la sustitución creciente de productos nacionales por bienes importados y la reorientación de la inversión directa de origen nacional hacia el comercio y los servicios, han provocado una tendencia sostenida hacia la terciarización del empleo» (Martínez 2006:5). Hubo una apertura importante de ese sector durante las décadas de 1960 y 1970, periodo en el que la población que ahora tiene más de 60 años de edad se encontraba en edad productiva.

Este proceso ha sido acompañado de un incremento del trabajo no asalariado, del crecimiento de pequeñas unidades económicas y de la precariedad laboral, elementos que se hacen evidentes dentro de la vida de los sujetos entrevistados no solo en cuanto a su trayectoria laboral, sino también por las actuales actividades que conservan o desarrollan para sobrevivir, donde hay vendedores de dulces, trabajadores y comerciantes por su cuenta y pocos empleados asalariados que gocen de seguridad social, como es el caso de El Chino (62 años), que se dedica a atender el centro social de su colonia, donde su remuneración son las propinas que le dan por limpiar y dejar entrar al baño a los tianguistas. ${ }^{5} \mathrm{~A}$ la pregunta de si tiene mucho trabajando ahí respondió de la siguiente manera:

- ¿Qué me quedaba? A esto le llaman trabajo

- Pues a fin de cuentas si le pagan es trabajo, ¿no?

- No me pagan, ahora está difícil encontrar un trabajo que te paguen, más a la edad de uno.

5 El vocablo se refiere a las personas que trabajan en un tianguis, españolización del náhuatl tianquiztli o tianquisco, que significa 'mercado'. La particularidad de un tianguis es que el comercio se realiza de manera itinerante, ya que no tiene un espacio o lugar permanente, sino que solamente se presenta uno o dos días a la semana. 
El Chino es un ejemplo recurrente de la realidad de Cuautitlán, donde al caminar por las calles de una de las colonias «viejas», como las llama la gente, el tianguis deja ver los rostros arrugados y las cabelleras blancas de las personas que atienden sus puestos en las calles, donde siempre — dicen ellos- se han dedicado a esto, a empleos o autoempleos que carecen de un salario y de seguridad social y, en consecuencia, no tienen acceso a jubilaciones, pensiones, amparo de instituciones de salud ni a créditos para la adquisición de vivienda. El testimonio de El Chino caracteriza estas condiciones de trabajo u ocupación de un sector que, comparado con otros grupos, vive en desventaja evidente (Wong et al. 2007) y que por su adscripción al autoempleo o al empleo informal (Ham 1999) pareciera tener como consecuencia lógica la precariedad en el acceso a la infraestructura de salud y seguridad social, lo cual contribuye, sin duda, a la vulnerabilidad de la población.
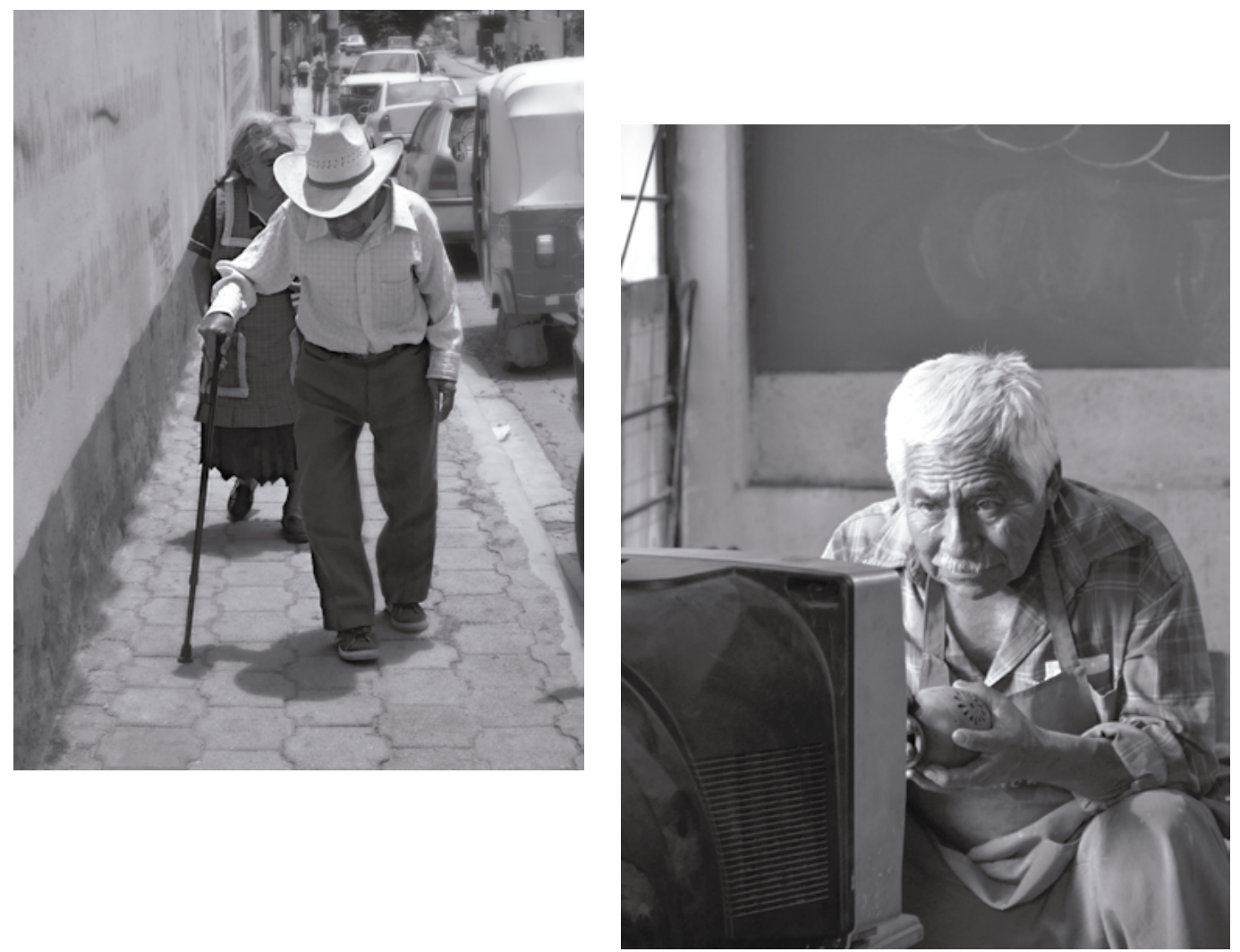

En cuanto al perfil de la población que recibe beneficios de las políticas públicas vigentes, uno de los factores que benefician potencialmente a las mujeres es la seguridad social por viudez, lo que implica recibir una pensión vitalicia aun 
sin haber mantenido una trayectoria laboral, es decir, reciben una pensión no contributiva.

Otra particularidad de los apoyos promovidos por el Estado, entre los que se encuentran los destinados a la ayuda alimentaria, es el criterio parcial con el que se decide la distribución o asignación de estos programas, ya que se destinan sobre todo a zonas rurales o campesinas, y se deja mayormente desprotegidos a los municipios urbanos que, como ya se vio en este trabajo, resultan ser los que alojan a más de $80 \%$ de los mexiquenses. Esta última circunstancia refleja una de las principales limitaciones de estos programas, ya que excluyen a la mayor parte de la población de más de 60 años, y esta ve reducido el apoyo a los programas reseñados en el cuadro 1. De ese modo, por ejemplo, los «paseos recreativos» están diseñados para personas con un ingreso seguro, así como con capacidades físicas —atendidas por su institución de salud-que le permiten realizar dicha actividad.

Don Vicente, de Tlalnepantla, con 65 años, dice respecto a los apoyos:
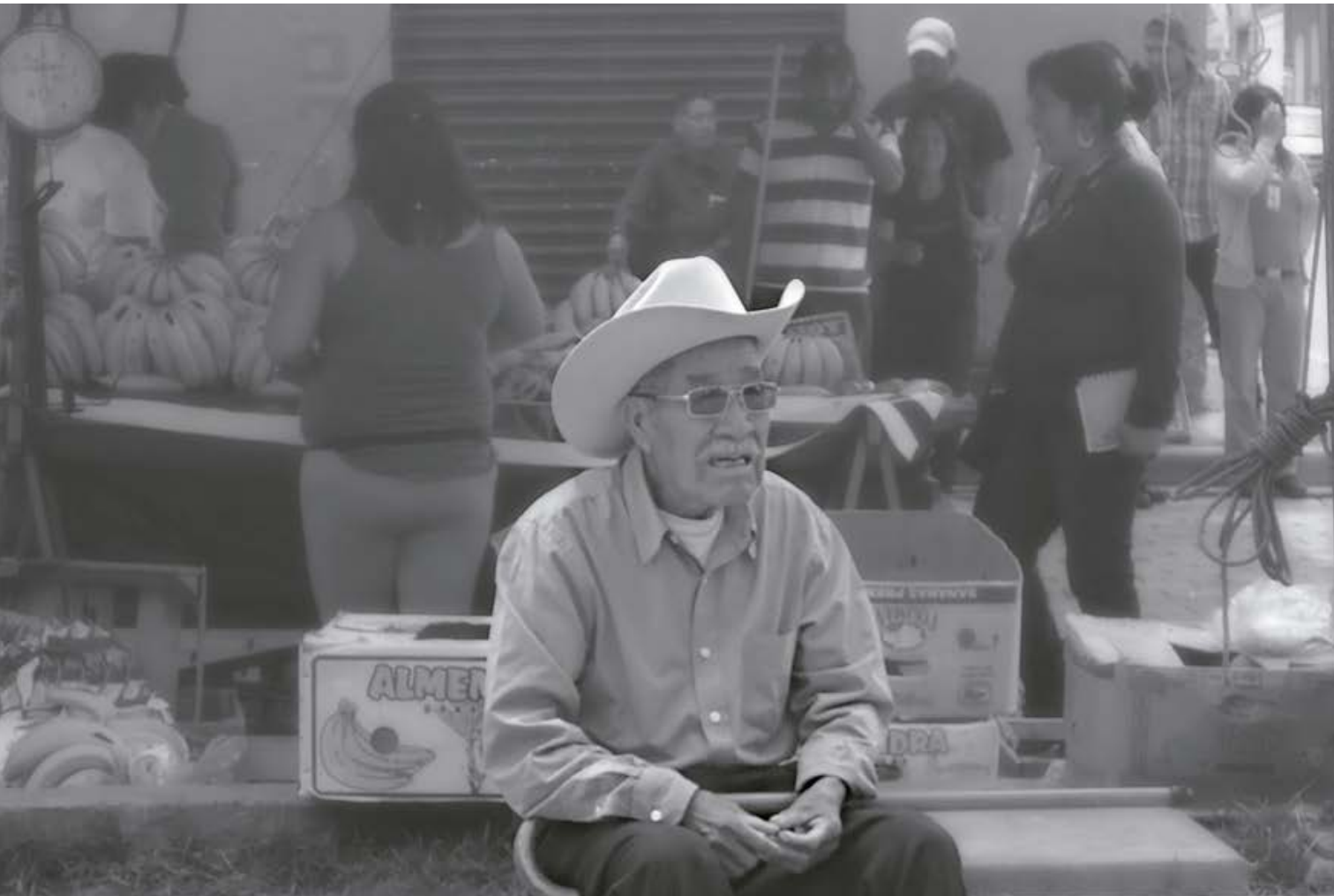
- Yo no quiero nada, a esos que les dan dinero nomás los hacen huevones, conformistas, que se pongan a trabajar, yo toda la vida he trabajado.

— ¿Qué cree, entonces, que le hace falta a las personas de más de 60 años?

- Trabajo

- ¿De plano?

- Sí, ¿de qué le sirven a uno 500 o 700 pesos?, yo lo que quiero es trabajo, que se pongan a trabajar los políticos, cuando uno vota por ellos la finalidad es que le den trabajo al mismo pueblo.

Don Leonardo, de 71 años, habitante de Temascalcingo, opina:

- No estoy de acuerdo que den dinero, está bien que den comida, porque el dinero yo ahorita agarro y me los juego en el billar, la comida es para todos.

En cuanto a las condiciones sociales y económicas en las que vive la población de más de 60 años, nuevamente se hace evidente la disparidad en los montos de las pensiones, una diferenciación que radica principalmente en las trayectorias laborales, pues las más elevadas (de hasta 15000 pesos) corresponden al grupo de los municipios con mayor índice de envejecimiento, debido a que algunos llegan a cotizar durante 40 o 50 años, mientras que en aquellos con mayor índice de derechohabiencia y los conurbados (Toluca, Metepec, Tlalnepantla, Cuauhtitlán, Ixtapaluca y Valle de Chalco) las pensiones son más bajas, y se cuenta como una menor cobertura de programas sociales, por lo que la relación que se expresa es que la población urbana tiene más carencias que la rural. Lo dicho queda de manifiesto en el siguiente testimonio:

—A los ocho años yo ya trabajaba allá en mi pueblo, iba a las cantinas, llenaba los tarros con cerveza... y acarrear agua y todo eso. Después ya me vine, pues, para poder salir de mi pueblo, porque en mi pueblo si no eres campesino tienes que ser minero... no hay de otra. Entonces tiene que buscar uno la salida, bueno, el que quiere, si no, uno ahí está clavado, y este, pues yo desesperadamente buscaba cómo salir de mi pueblo.

- ¿Desde entonces trabaja?

-Desde entonces, nunca he dejado de trabajar, y espero no tener que hacerlo. 
Don Wenseslao, de 65 años y habitante de Tlalnepantla opina si ya se quiere retirar, después de 38 años laborando en el área de hojalatería, en la industria automotriz:

- No, no, no, no...porque mi idea es seguir trabajando los más años que pueda... mientras me den trabajo

- Pero, ¿usted no quiere dejar de trabajar?

- La verdad, no, ¿eh?

Otra línea de la investigación concierne a una distinción en las actividades que desempeñaron hombres y mujeres. Si bien ellos se desenvolvieron entre el campo y la industria, en las zonas rurales era común que ellas se dedicaran a trabajos en el sector de los servicios. Así se constata dentro de los municipios mayormente envejecidos, que son poblaciones pequeñas, donde las mujeres empezaron una trayectoria laboral temprana en la docencia, respondiendo a las necesidades sociales del momento histórico; sus pensiones, que oscilan entre 7000 y 20000

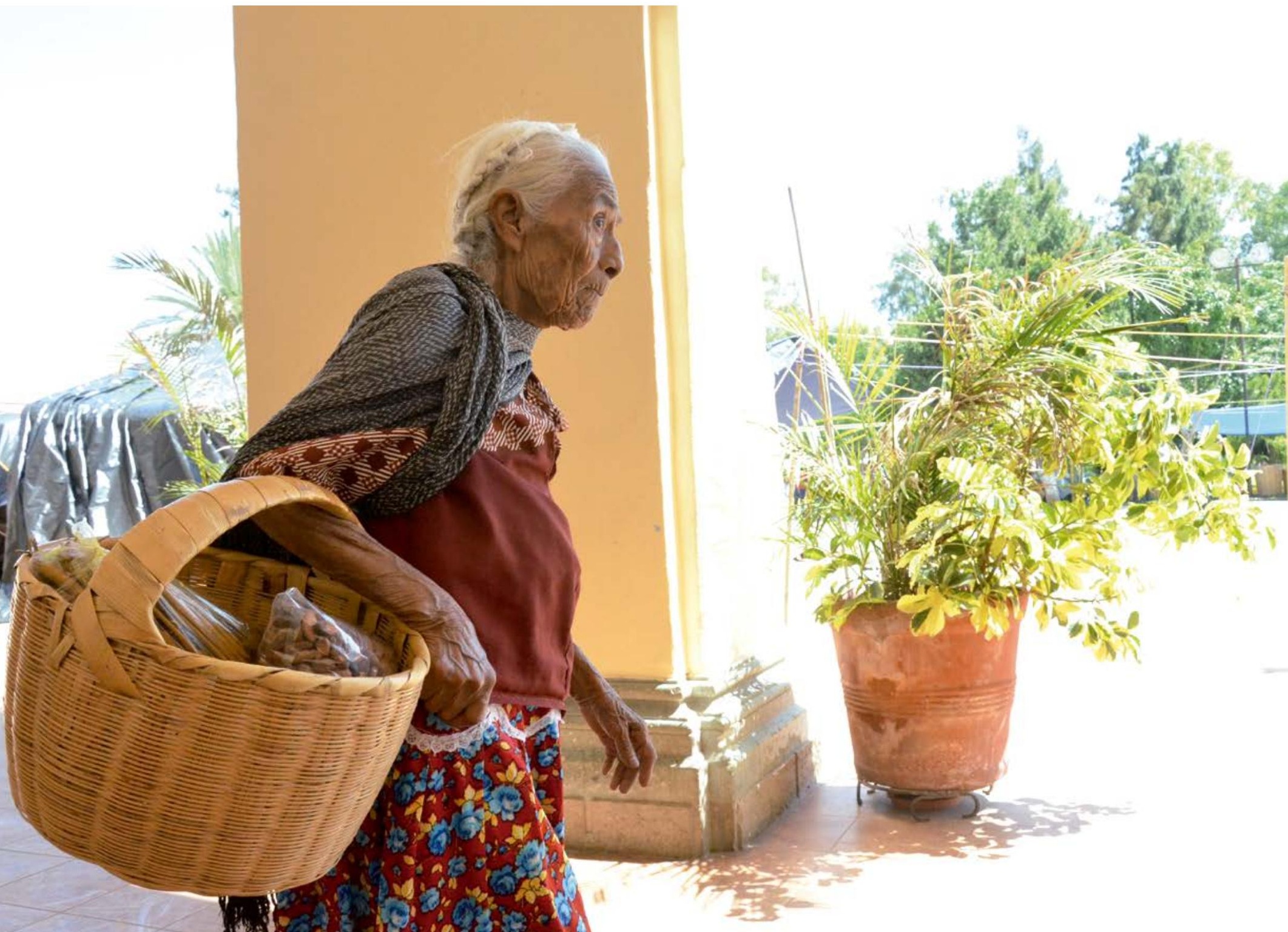


pesos al mes, son una muestra de que los programas sociales no son distribuidos de una manera eficiente. Al respecto, don Desiderio, de 72 años, habitante de Tejupilco, opina de la asignación de los programas sociales:

-Pues yo creo que deberían ver a quién se los dan, porque acá abajo hay una maestra, bueno, que ya no trabaja, pero fue maestra aquí siempre, y le dan su despensa, yo creo que eso no está bien, porque hay gente que de veras necesita esas cosas.

La población envejecida de la entidad se expresa así en relación con un tema tan importante y deja ver la necesidad de replantear no solo ejecución de los programas sociales en el estado, sino analizar desde el origen y la creación de estos programas que no se han basado en la realidad social.

\section{A MANERA DE CONCLUSIÓN LA INTENCIÓN DEL TRABAJO ANTROPOLÓGICO}

Una perspectiva especialmente relevante en los trabajos antropológicos en torno a la vejez es aquella que resalta el envejecimiento como una problemática social y cultural.

En este trabajo se considera importante resaltar el hecho de que si bien la población envejecida ha tomado un lugar dentro de las políticas públicas vigentes en la entidad, lo cual no sucedía hace siquiera 10 años, no debe considerarse como un logro o una solución a la problemática social que vive la población de más de 60 años de edad y que se incrementará en las próximas dos décadas, por lo que es necesario replantearnos la pregunta que se hiciera Foster hace casi 50 años: «¿Qué es lo que, en la ciencia de la antropología, es pertinente en la búsqueda de soluciones a los problemas contemporáneos de cambio social?» (1974[1969]:9), lo que nos lleva a pensar en que la antropología ofrece la oportunidad de precisar necesidades de la población en estudio, en este caso a partir de la percepción de personas que reclaman la creación de políticas públicas que recaigan directamente en la creación de programas de incorporación laboral del grupo etario de 60 y más años de edad de manera integral, partiendo de lo que Laurel (1997) dice al respecto, pues «el concepto de integralidad del sistema de seguridad social corresponde a la idea de que debe proporcionar un conjunto de beneficios y servicios sociales lo suficientemente amplio como para garanti- 
zar condiciones de vida razonables y el desarrollo pleno de los seres humanos» (Laurel 1997:52).

Evidentemente, no hay una clara relación entre la asignación de apoyos a la población envejecida y su realidad; asimismo, es cuestionable el formato actual de los programas sociales; en consecuencia, se vuelve necesario el paso de la antropología teórica a la aplicada, donde se participe en la creación de nuevas políticas a partir de la realidad de los sujetos, y las necesidades expresadas sean la guía de nuevos criterios para establecer programas públicos asistenciales, contribuyendo así a la estabilidad de la sociedad que envejece.

Si se afirma que los programas sociales en el Estado de México son asistencialistas es porque, en la visión de Huenchuan (2003), sus acciones parecen destinadas a la supervivencia de la población envejecida. Esta, a su vez, expresa la necesidad de solucionar de raíz sus problemas, es decir, resolver la falta de trabajo, el difícil acceso a los servicios de salud, la precariedad de los ingresos tanto en zonas urbanas como rurales. Por otro lado, opinan que no es clara la designación de los beneficiarios de los programas, o no están dirigidos a las personas que necesitan el recurso otorgado, de ahí la insistencia en llamarlos asistencialistas.

Y aunque, como afirma Laurel (1997) «los principios fundantes de la seguridad social mexicana son la integralidad y la amplitud de los servicios-beneficios sociales: la solidaridad y la redistribución de recursos, además del carácter obligatorio y publico del aseguramiento», sin embargo, en la realidad social no son evidentes ni están cerca de serlo para la población envejecida. El desarrollo de esta investigación afirma la necesidad de conocer aspectos de un grupo de población que necesita la creación de políticas públicas adecuadas, pertinentes y eficaces.

\section{FUENTES DE CONSULTA}

Aguirre Beltrán, Gonzalo, 1955, Obra antropológica, V. Programas de salud en la situación intercultural, México, Fondo de Cultura Económica.

Aguirre Beltrán, Gonzalo, 1973, Obra antropológica, X. Teoría y práctica de la educación indígena, México, FCE.

Aranibar, Paula, 2001, Acercamiento conceptual a la situación del adulto mayor en América Latina, Serie Población y Desarrollo, 21, Santiago de Chile, CEPAL

Ávalos Pérez, Rosaura, 2005, Aproximaciones a las repercusiones en la unidad doméstica de la pensión alimentaria para adultos mayores de 70 años, residentes en el D. F., tesis de maestría inédita, México, CIESAS. 
Bonfil Batalla, Guillermo, 1991, México profundo. Una civilización negada, México, Alianza Editorial.

Caso, Alfonso, 1948, «Definición del indio y de lo indio», América Indígena, 8(4), pp. 239-247.

Centro Nacional de Desarrollo Municipal, 1997, Resumen estatal de la reglamentación municipal del Estado de México, México, CEDEMUN.

Consejo Nacional de Población, 2012, <http://www.conapo.gob.mx/> [consulta: 10 de enero de 2012, 10 de agosto de 2014].

Foster, George, 1974[1969], Antropología aplicada, México, Fondo de Cultura Económica (Breviarios).

Ham Chamde, Roberto, 1999, «El envejecimiento en México: de los conceptos a las necesidades», Papeles de Población, Nueva época, 5(19), pp. 7-21.

Huenchuan Navarro, Sandra, 2003, Políticas de Vejez en América Latina. Una propuesta para su análisis, 51, Congreso internacional de Americanistas, Santiago de Chile.

Instituto Nacional de Estadística y Geografía (INEGI), 2011, "Censos y conteos de población y vivienda» <http://www.inegi.org.mx/sistemas/mexicocifras/default.aspx? $=15>$ [consulta: 20 de enero de 2012.].

Laurel, Asa Cristina, 1997, La reforma contra la salud y la seguridad social: una mirada crítica y una propuesta alternativa, México, Ediciones ERA.

López Paniagua, Rosalía, 2004, Pobreza Urbana y neoliberalismo en México. Formas de acceso a la vivienda y alternativas de política social, México, Universidad Nacional Autónoma de México.

Martínez de la Peña, Mónica, 2006, «Mercado de trabajo y participación económica familiar en Monterrey, 1990-2003», tesis de doctorado inédita, México, El Colegio de México.

Menéndez, Eduardo, 1999, «Uso y desuso de los conceptos. ¿Dónde quedaron los olvidos?», Alteridades, 9(17), pp. 149-167.

Reyes Gómez, Laureano, 1999, «Salud, enfermedad y atención en la vejez», tesis de doctorado inédita, Tijuana, El Colegio de la Frontera Norte.

Secretaría de Finanzas y Planeación, 1995, «Enciclopedia de los Municipios», Toluca, México, <http://www.e-local.gob.mx/wb2/ELOCAL/ELOC> [consulta: 10 de enero de 2012].

Vázquez Palacios, Felipe 1999, «Hacia una cultura de la ancianidad», Papeles de población, Nueva época, 5(19), pp. 65-75.

Wong, Rebeca, Mónica Espinoza, Alberto Palloni, 2007, «Adultos mayores mexicanos en contexto socioeconómico amplio: salud y envejecimiento» Salud Pública de México, 49, suplemento 4, pp. 436-447.

Fecha de recepción: 24 de noviembre de 2015

Fecha de aceptación: 8 de marzo de 2016 